\title{
Study on the Tensile Performance of PTT Fiber
}

\author{
Yuanyuan Zheng \\ Composites Institute \\ Tianjin Polytechnic University \\ Tianjin 300160, China \\ E-mail: yuanyuanzheng1983@163.com \\ Jialu Li \\ Composites Institute \\ Tianjin Polytechnic University \\ Tianjin 300160, China \\ Ruizhou Li \\ Textile and Apparel Institute \\ Hebei University of Technology \\ Shijiazhuang 050018, China
}

\begin{abstract}
To deeply study the mechanics performance of PTT fiber and develop the application domain of PTT fiber, in this article, we implement comparative research of tensile performance to PTT fiber and PET fiber respectively in wet condition and dry condition through experiments, and indicate the advantage of PTT fiber. And based on the experiment result, we study the tensile performance of PTT fiber after acid disposal and alkali disposal, which indicates the acid and alkali resistance of PTT fiber and establishes the base to better be applied in the actual production for PTT fiber.
\end{abstract}

Keywords: PTT, PET, Tensility, Acid resistance, Alkali resistance

\section{Introduction}

PTT fiber is a sort of new poalkalister material, and it is assembled by terephthalic acid (TPA) and 1,3-propandiol (PDO), and the homolog of poalkalithylene terephthalate (PET) and poly butylene terephthalate (PBT), and it has many advantages of PET fiber, PBT fiber and PA fiber, such as good elasticity, plasticity, chromaticity, and low pollution to the environment, and it is called as the large fiber in the 21 st century (Chen, 2005, p.7-12).

With the extensive use of PTT fiber in the spinning industry, it is urgent to research its performances, especially the tensile performance of PTT fiber. Based on many experiments, in this article, we analyze and study the tensile performance of PTT fiber in four conditions including dry condition, wet condition, acid condition and alkali condition, which possesses certain directional meanings for the spinning technology.

\section{Experiment}

\subsection{Sample}

The sample is the PTT fiber with the length of $38 \mathrm{~mm}$ and the fiber fineness of $1.67 \mathrm{dtex}$.

\subsection{Instrument}

The instrument in the experiments is the XQ-1 fiber strength elongation instrument.

\subsection{The tensile experiment of PTT fiber in dry condition and wet condition}

The testing conditions conclude that the distance between gripper is $10 \mathrm{~mm}$, the pre-tensile force is $0.1 \mathrm{cN}$, the tensile speed is $10 \mathrm{~mm} / \mathrm{min}$, and sample amount is 50 pieces, and the experiment results are seen in Table 1.

\subsection{Acid resistance performance experiment of PTT fiber}

\subsubsection{Experiment reagent and instrument}

The Experiment reagent and instruments include oil of vitriol, beaker, measuring cylinder, thermometer, scale, constant temperature water bathing pan, glass stick and constant temperature oven. 


\subsubsection{Experiment scheme}

Respectively put up 5\%,20\%,40\% and $60 \%$ vitriol liquors, and do the experiment once when the temperature raises $30{ }^{\circ} \mathrm{C}$ every time from $30^{\circ} \mathrm{C}$ to $90^{\circ} \mathrm{C}$, and every temperature is kept in $30 \mathrm{~min}, 60 \mathrm{~min}$ and $90 \mathrm{~min}$, and in every group, record 20 data and compute the average. The bathing ratio is 1:20, and the experiment results are seen in Table 2, Table 3 and Table 4.

\subsection{Alkali resistance performance experiment of PTT fiber}

\subsubsection{Experiment reagent and instrument}

The Experiment reagent and instruments include solid sodium hydroxide, beaker, measuring cylinder, thermometer, scale, constant temperature water bathing pan, glass stick and constant temperature oven.

\subsubsection{Experiment scheme}

Respectively put up 5\%,15\% and 25\% sodium hydroxide liquors, and do the experiment once when the temperature raises $20^{\circ} \mathrm{C}$ every time from $30^{\circ} \mathrm{C}$ to $70^{\circ} \mathrm{C}$, and every temperature is kept in $15 \mathrm{~min}, 30 \mathrm{~min}$ and $45 \mathrm{~min}$, and in every group, record 20 data and compute the average. The bathing ratio is 1:20, and the experiment results are seen in Table 5, Table 6 and Table 7.

\section{Result analysis of experiment}

(1) From Table 1, we can know that the tensile degree of PTT fiber is high in the dry condition, and its breaking mightiness can achieve $8.7 \mathrm{cN}$ maximally, and its breaking tensile ratio can maximally achieve $81.3 \%$, but in the wet condition, both the fiber breaking mightiness and the breaking tensile ratio reduce, and whether in the dry condition and in the wet condition, the breaking mightiness of PTT fiber is lower than PET fiber, but its breaking tensile ratio is far higher than PET fiber.

The reason is that the PTT fiber possesses the zigzag molecule structure which is easier to produce distortion, so the ability to resist the tensile and destroying of outside force is high, but the big molecule chain in the PET fiber almost presents a structure with complete stretch, only little stretch can completely extend the molecule, so the initial modulus of PET is higher, and the tensile recover elasticity is bad, and because the curve rigidity and the pliability of fiber are influenced by the initial modulus, so PTT fiber possesses high elasticity and good elasticity recovery ratio, and the fabric made by PTT fiber has better pliability than PET fiber.

(2) From Table 2, Table 3 and Table 4, we can know that, when the temperature is in $30^{\circ} \mathrm{C}$, the breaking mightiness and the breaking tensile ratio of PTT after acid disposal change little, and with the increase of vitriol concentration, the breaking tensile ratio goes to increase, and when the temperature is in $60^{\circ} \mathrm{C}$, the breaking intension and the breaking tensile ratio of PTT obviously reduce with the increase of vitriol concentration, but the influence of time to the breaking mightiness is not obvious, and the breaking tensile ratio gradually reduces with the prolonging of water bathing time, and when the temperature is in $90^{\circ} \mathrm{C}$, two factors influence the PTT fiber breaking mightiness and breaking tensile ratio very significantly, and the vitriol concentration is higher and the disposal time is longer, the breaking mightiness and the breaking tensile ratio reduce more obviously.

The breaking mightiness and the breaking tensile ratio of PTT fiber gradually reduce with the increase of temperature, the increase of vitriol concentration and the prolonging of disposal time, and its change ranges are $0 \sim 60 \%$ of breaking mightiness and $0 \sim 28 \%$ of breaking tensile ratio.

(3) From Table 5, Table 6 and Table 7, we can know that the breaking mightiness and the breaking tensile ratio of PTT fiber after alkali liquors in different temperatures and different time period change little, and its breaking tensile increases little on the contrary, and its change ranges $0 \sim 24 \%$ of breaking mightiness and $0 \sim 58 \%$ of breaking tensile ratio.

\section{Conclusions}

The mightiness of PTT fiber is in the cotton fiber and the terylene fiber, and it is higher than cotton fiber, so it is can enhance the breaking of mixed spanning when PTT fiber is mixed with cotton fiber. The breaking mightiness is lower $20 \% \sim 30 \%$ than terylene fiber, but its breaking tensile ratio is far higher than terylene fiber, so the PTT fiber possesses excellent tensile recovery performance. PTT fiber can resist alkali but not acid, so we should pay attention to this point when selecting the fiber or in the use process of PTT pure spinning and mixed spinning fabrics.

\section{References}

Chen, Kequan. (2005). Development of PTT Products and its Commercial Production in Shanghai Petrochemical Co., Ltd. Synthetic Fiber in China. No.2. p.7-12.

Fang, Xuejuan. (2005). The Structure, Property and Application of PTT Fiber and Fabric. Synthetic Fiber in China. No.2. p.32-34. 
Liu, Qiuying \& Zhang, Youhe. (2005). Development of Novel Ring-spun Yarns, Lecture 5 Properties of the New Type Poalkalister Fiber-PTT and the Textile Production Technology. Advanced Textile Technology. No.5. p.54-56.

Qiu, Yufa. (2005). Product Development with PTT Fiber. Advanced Textile Technology. No.6. p.35-36.

S. Deiss \& E. Seidel. (2005). Continuous PTT Process. Synthetic Fiber in China. No.2. p.41-44.

Wei, Gaofu, Gu, Lixia, Dai, Zhibin \& Changhua. (2005). The Research Advance of Poly(trimethylene terephthalate). Synthetic Fiber in China. No.2. p.1-6.

Wei, Gaofu. (2005). Synthetic Research on PTT. Synthetic Fiber in China. No.2. p.20-22.

Table 1. The tensile experiment results of PTT fiber in the dry or wet condition

\begin{tabular}{|c|c|c|c|c|}
\hline \multirow{2}{*}{ Item } & \multicolumn{2}{|c|}{ Dry condition } & \multicolumn{2}{c|}{ Wet condition } \\
\cline { 2 - 5 } & BF (cN) & ET (\%) & BF (cN) & ET (\%) \\
\hline PTT & 5.50 & 50.40 & 5.16 & 46.00 \\
\hline PET & 8.50 & 33.04 & 8.40 & 32.30 \\
\hline
\end{tabular}

Table 2. The acid resistance of PTT fiber in the water bathing temperature of $30^{\circ} \mathrm{C}$

\begin{tabular}{|c|c|c|c|c|c|c|c|c|}
\hline \multirow{2}{*}{ Item } & \multicolumn{2}{|c|}{$5 \%$} & \multicolumn{2}{c|}{$20 \%$} & \multicolumn{2}{c|}{$40 \%$} & \multicolumn{2}{c|}{$60 \%$} \\
\cline { 2 - 9 } & BF (cN) & ET (\%) & BF (cN) & ET (\%) & BF (cN) & ET (\%) & BF (cN) & ET (\%) \\
\hline $30 \mathrm{~min}$ & 5.0 & 85.3 & 5.0 & 87.7 & 5.2 & 89.5 & 5.0 & 70.6 \\
\hline $60 \mathrm{~min}$ & 5.0 & 74.1 & 5.1 & 82.2 & 5.5 & 84.6 & 5.6 & 89.6 \\
\hline $90 \mathrm{~min}$ & 5.2 & 84.3 & 5.1 & 82.2 & 5.0 & 84.0 & 5.1 & 86.5 \\
\hline
\end{tabular}

Table 3. The acid resistance of PTT fiber in the water bathing temperature of $60^{\circ} \mathrm{C}$

\begin{tabular}{|c|c|c|c|c|c|c|c|c|}
\hline \multirow{2}{*}{ Item } & \multicolumn{2}{|c|}{$5 \%$} & \multicolumn{2}{c|}{$20 \%$} & \multicolumn{2}{c|}{$40 \%$} & \multicolumn{2}{c|}{$60 \%$} \\
\cline { 2 - 9 } & BF (cN) & ET (\%) & BF (cN) & ET (\%) & BF (cN) & ET (\%) & BF (cN) & ET (\%) \\
\hline $30 \mathrm{~min}$ & 5.3 & 87.8 & 5.1 & 92.5 & 4.8 & 89.0 & 4.4 & 68.6 \\
\hline $60 \mathrm{~min}$ & 5.4 & 78.7 & 5.5 & 78.4 & 5.5 & 79.1 & 4.6 & 65.6 \\
\hline $90 \mathrm{~min}$ & 5.2 & 87.6 & 5.6 & 84.2 & 5.4 & 81.3 & 4.1 & 61.6 \\
\hline
\end{tabular}

Table 4. The acid resistance of PTT fiber in the water bathing temperature of $90^{\circ} \mathrm{C}$

\begin{tabular}{|c|c|c|c|c|c|c|c|c|}
\hline \multirow{2}{*}{ Item } & \multicolumn{2}{|c|}{$5 \%$} & \multicolumn{2}{c|}{$20 \%$} & \multicolumn{2}{c|}{$40 \%$} & \multicolumn{2}{c|}{$60 \%$} \\
\cline { 2 - 9 } & BF (cN) & ET (\%) & BF (cN) & ET (\%) & BF (cN) & ET (\%) & BF (cN) & ET (\%) \\
\hline $30 \mathrm{~min}$ & 5.2 & 89.4 & 5.4 & 88.3 & 4.9 & 74.2 & 3.0 & 43.9 \\
\hline $60 \mathrm{~min}$ & 5.3 & 78.2 & 4.8 & 71.9 & 5.3 & 88.5 & 2.6 & 38.2 \\
\hline $90 \mathrm{~min}$ & 5.1 & 94.1 & 4.7 & 80.8 & 4.6 & 69.6 & 2.2 & 36.3 \\
\hline
\end{tabular}


Table 5. The alkali resistance of PTT fiber in the water bathing temperature of $30^{\circ} \mathrm{C}$

\begin{tabular}{|c|c|c|c|c|c|c|}
\hline \multirow{2}{*}{ Item } & \multicolumn{2}{|c|}{$5 \%$} & \multicolumn{2}{c|}{$15 \%$} & \multicolumn{2}{c|}{$25 \%$} \\
\cline { 2 - 7 } & BF (cN) & ET (\%) & BF (cN) & ET (\%) & BF (cN) & ET (\%) \\
\hline $15 \mathrm{~min}$ & 4.5 & 71.5 & 4.2 & 69.7 & 4.3 & 70.3 \\
\hline $30 \mathrm{~min}$ & 4.7 & 73.5 & 4.4 & 70.6 & 5.1 & 82.1 \\
\hline $45 \mathrm{~min}$ & 4.6 & 79.4 & 4.7 & 68.2 & 4.3 & 75.6 \\
\hline
\end{tabular}

Table 6. The alkali resistance of PTT fiber in the water bathing temperature of $50^{\circ} \mathrm{C}$

\begin{tabular}{|c|c|c|c|c|c|c|}
\hline \multirow{2}{*}{ Item } & \multicolumn{2}{|c|}{$5 \%$} & \multicolumn{2}{c|}{$15 \%$} & \multicolumn{2}{c|}{$25 \%$} \\
\cline { 2 - 7 } & BF (cN) & ET (\%) & BF (cN) & ET (\%) & BF (cN) & ET (\%) \\
\hline $15 \mathrm{~min}$ & 4.7 & 73.8 & 4.5 & 67.5 & 4.7 & 76.7 \\
\hline $30 \mathrm{~min}$ & 4.9 & 79.7 & 4.6 & 72.3 & 4.2 & 69.2 \\
\hline $45 \mathrm{~min}$ & 4.9 & 75.3 & 4.4 & 69.8 & 4.3 & 70.3 \\
\hline
\end{tabular}

Table 7. The alkali resistance of PTT fiber in the water bathing temperature of $70^{\circ} \mathrm{C}$

\begin{tabular}{|c|c|c|c|c|c|c|}
\hline \multirow{2}{*}{ Item } & \multicolumn{2}{|c|}{$5 \%$} & \multicolumn{2}{c|}{$15 \%$} & \multicolumn{2}{c|}{$25 \%$} \\
\cline { 2 - 7 } & $\mathrm{BF}(\mathrm{cN})$ & $\mathrm{ET}(\%)$ & $\mathrm{BF}(\mathrm{cN})$ & $\mathrm{ET}(\%)$ & $\mathrm{BF}(\mathrm{cN})$ & ET (\%) \\
\hline $15 \mathrm{~min}$ & 4.7 & 78.5 & 4.0 & 59.4 & 4.7 & 75.1 \\
\hline $30 \mathrm{~min}$ & 4.6 & 78.0 & 4.7 & 74.3 & 4.4 & 74.8 \\
\hline $45 \mathrm{~min}$ & 4.2 & 66.0 & 4.4 & 68.8 & 4.2 & 64.1 \\
\hline
\end{tabular}

